\title{
Nota Técnica: \\ Análisis estadístico de los defectos de juntas cerámicas
}

\author{
Technical Note: \\ Statistical analysis of defects \\ of tiles" joints
}

$\underline{\text { J. Silvestre }}^{(*)}$ y J. de Brito(**)

Recepción/Received: 23-XII-05

Aceptación/Accepted: 4-VII-06

\section{RESUMEN}

En este trabajo se analizan los defectos de junta que pueden aparecer en las distintas aplicaciones cerámicas actuales. En primer lugar, se propone una clasificación tanto de los defectos como de las causas más probables de los mismos. A continuación se presenta una matriz de correlación de dichos defectos y causas probables.

A partir de 88 inspecciones de paramentos cerámicos, se han identificado los defectos del material de juntas más frecuentes, describiéndose los resultados del análisis estadístico de los datos recogidos con motivo de dichas inspecciones. En los cuadros correspondientes se especifican la frecuencia del defecto, las causas observadas y la relación entre ambas en la muestra.

Los datos recogidos in situ evidencian una alta incidencia de defectos del material de juntas en esta clase de soluciones cerámicas. Dicha conclusión constata la necesidad de actuaciones preventivas, que deberían incluir el correcto diseño de las juntas cerámicas y la planificación previa de los trabajos de mantenimiento (con visitas periódicas y tareas de reparación). Se sostiene que la única forma de conseguir que la durabilidad del revestimiento cerámico sea la prevista es mediante un mantenimiento adecuado.

Palabras clave: patologías, ladrillo, cerámica, mortero, deterioro.

\section{SUMMARY}

The present article addresses tile joint defects in interior and exterior floors and walls. It begins with a classification of tile joint defects along with their likely causes, establishing a correlation matrix between the two sets of parameters. This is followed by the identification of the flaws found most frequently in this type of cladding, based on field data collected during 88 inspections of tile joint material.

The statistical analysis of these data is shown in the form of charts representing the frequency of joint defects, their causes and the interrelationships between the two.

The field work confirmed the high frequency of joint defects in all types of tiling. This conclusion underscores the need for proper joint design and maintenance planning (including periodic inspection and repair as appropriate) to guarantee the durability of this type of cladding.

Keywords: pathology, tile, ceramic, mortar, deterioration.

\footnotetext{
(*) Instituto Técnico de Lisboa / Laboratorio Nacional de Ingeniería Civil, Lisboa (Portugal).

(**) Instituto Técnico de Lisboa (Portugal).
}

Persona de contacto / Corresponding author: joseds@netvisao.pt 


\section{INTRODUCCIÓN}

El material de juntas es el componente del revestimiento cerámico más susceptible frente a los agentes agresivos, que también inciden en la estabilidad del conjunto. En este trabajo, se identifican y describen los distintos defectos que se presentan en el material de juntas, así como sus causas más probables. Según los datos recogidos in situ, la frecuencia de defectos del material de juntas es alta en un número significativo de los casos estudiados.

En el análisis estadístico de dichos datos se ha contemplado asimismo la frecuencia de las causas observadas.

\section{DEFECTOS DEL MATERIAL DE JUNTAS}

La clasificación de defectos del material de juntas propuesta incluye todos los que pueden afectar al revestimiento cerámico, incluyendo los estéticos. Dicha clase de defectos, como un cambio de color, por ejemplo, puede llevar a una pérdida de las características iniciales del material de juntas o incluso de las prestaciones del material cerámico.

Un solo defecto del material de juntas puede permitir que el agua se filtre por debajo del mortero de agarre en puntos localizados, provocando el deterioro de la junta y reduciendo la durabilidad del revestimiento cerámico, proceso que será tanto más rápido cuanto más agresivo sea el medio. En la Tabla 1 se presenta la clasificación de defectos de juntas con fotos representativas (1).

El deterioro de las juntas cerámicas puede producirse por muchas causas (2-4). Éstas pueden agruparse en los epígrafes habituales que se describen más adelante, como son los errores de diseño, de ejecución, la acción mecánica de agentes exteriores, las acciones ambientales, la falta de mantenimiento y el cambio de las condiciones iniciales. A fin de presentar las causas de los defectos del revestimiento cerámico en orden cronológi$\mathrm{Co}$, se ha propuesto un sistema de clasificación de las mismas (5). De entre todas estas causas, se han seleccionado las que interesan a las juntas, de manera directa o indirecta (Tabla 3).

\subsection{Errores de diseño (C-A)}

Durante la fase de proyecto del revestimiento cerámico, es necesario tomar decisiones importantes en relación con la selección del material, tales como el grosor y disposición de las juntas para evitar el deterioro provocado por la acción de agentes exteriores. Las prestaciones y durabilidad del material cerámico dependen del material de juntas elegido en cada caso $(6,7)$. En un revestimiento cerámico, las juntas suelen ser el único punto de salida que tiene el agua de condensación retenida en el material del soporte, dada la estanqueidad de la cerámica. El material de juntas también ha de tener una capilaridad baja a fin de impedir el transporte de sales solubles hacia la superficie para así evitar la formación de eflorescencias (8).

\subsection{Errores de ejecución (C-B)}

Se suele enlechar unas $24 \mathrm{~h}$ después de colocar las piezas cerámicas. Si no se realiza con esmero, esta fase puede dar origen a varios defectos. Una ejecución incorrecta, por falta de presión suficiente al aplicar la lechada, por ejemplo, o el empleo de una dosificación excesiva de cemento, podría provocar la formación de fisuras por retracción excesiva (9).

Tabla 1. Propuesta de clasificación de defectos de juntas

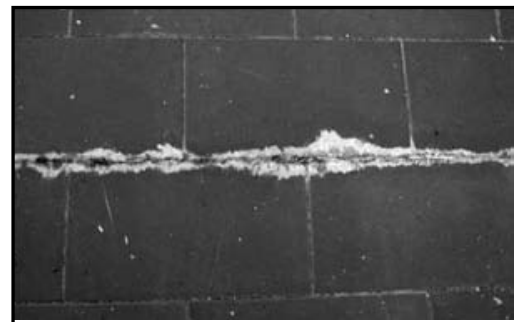

Dt.j 1 Eflorescencias

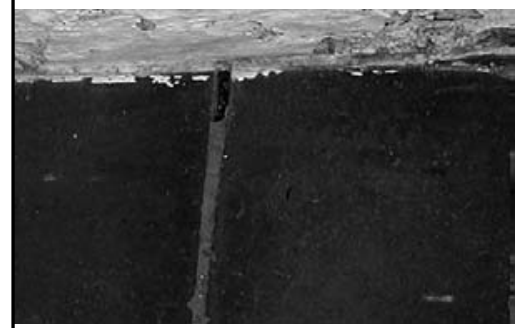

Dt.j 4 Desprendimiento

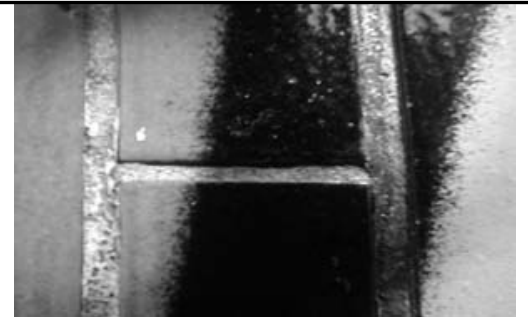

Dt.j 2 Cambio de color

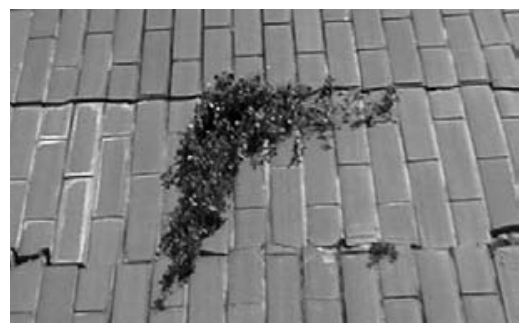

Dt.j 5 Biodeterioro

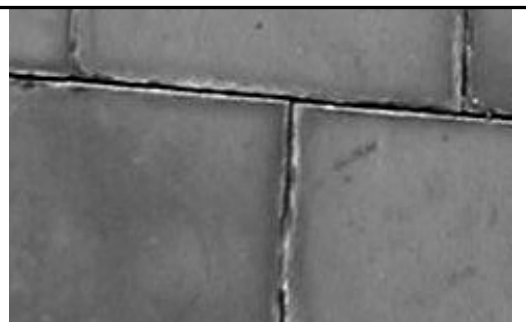

Dt.j 3 Agrietamiento

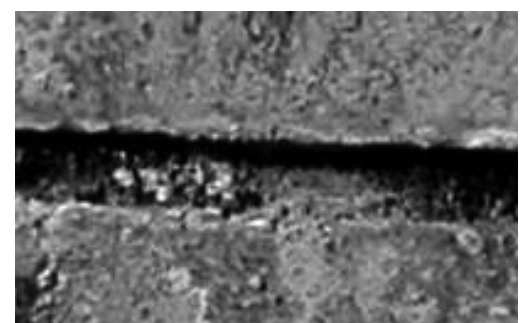

Dt.j 6 Pulverulencia 




Figura 1. Esquemas de enlechados: correcto (izquierda) e incompletos (centro y derecha) (10).

El esquema de la izquierda de la Figura 1 muestra un enlechado de juntas correcto. En el centro y a la derecha, el enlechado es incompleto, por falta de material en los extremos y por grosor insuficiente, respectivamente.

Se deben colocar las piezas de forma tal que las juntas sean rectas y regulares, y con un grosor variable de acuerdo con la clase y forma de la pieza y las cargas de servicio que ha de soportar. La lechada podrá aplicarse con la ayuda de separadores de plástico que aseguren la uniformidad del grosor de la junta. La superficie cerámica es practicable, normalmente, dos semanas después de la aplicación de la lechada, una vez fraguado el material de agarre (8).

\subsection{La acción mecánica de agentes externos (C-C)}

Este grupo de acciones aleatorias, que pueden afectar a cualquier tipo de revestimiento cerámico, son imposibles de prever o de prevenir de manera eficaz. De hecho, la prevención comienza en la fase de proyecto con la selección de la lechada, que debe realizarse en función de las características físico-químicas buscadas.

El graffiti es una acción a la que están expuestas las fachadas cerámicas, ya que su ubicación exterior hace de ellas un excelente escaparate para este género de arte. Un grupo de investigadores brasileños ha ensayado una serie de productos (un barniz y alguna silicona hidrófuga) para determinar su eficacia antigraffiti en paramentos cerámicos (11). Observaron que aun cuando las fachadas se habían protegido con dichos productos, al eliminar el graffiti no se conservaban ni el color ni el brillo de la cerámica. Por otra parte, se eliminaba el graffiti más fácilmente del material cerámico vidriado que de las juntas. En el presente trabajo, se han empleado disolventes y detergentes neutros para la limpieza de las fachadas cerámicas.

\subsection{Acciones ambientales (C-D)}

Al formar parte de la superficie del paramento, las juntas cerámicas se hallan directamente expuestas a todas las acciones ambientales. Si la humedad relativa del aire es alta al colocar el material cerámico, este puede desprenderse prematuramente o presentar eflorescencias a causa de la humectación de las sales solubles presentes en o debajo del material de agarre (12). Las eflorescencias pueden proceder también de la lixiviación del mortero de cemento del lecho o de la lechada, con lo que se produce la carbonatación del hidróxido de calcio presente en la superficie del material cerámico. En la Tabla 2 , se indica el origen de cada clase de eflorescencias, así como el sistema de limpieza recomendado.

El crecimiento de micro (algas, líquenes o musgos) o macro (vegetación) organismos sobre la superficie de las piezas o las juntas también puede ser motivo de la degradación del material cerámico. Este tipo de defecto es más habitual en paramentos exteriores situados en umbrías húmedas (12).

\subsection{Falta de mantenimiento (C-E)}

La durabilidad de un revestimiento cerámico se halla directamente influenciado por su utilización y mantenimiento. Por lo tanto, no puede suponerse que su durabilidad sea la del material cerámico empleado si se descuida el mantenimiento necesario (9). Un correcto mantenimiento permite identificar la fase de degradación en que se hallan el material cerámico y las juntas, y eliminar los microorganismos y otras sustancias indeseables mediante una limpieza adecuada. Por otra parte, la limpieza periódica de las juntas asegura su permeabilidad al vapor de condensación, característica que es esencial para el intercambio gaseoso (11). También pueden darse casos de limpieza del material cerámico con productos inadecuados como las sustancias fuertemente alcalinas o ácidas, o que tienen sulfatos en su composición. Dichos productos

Tabla 2. Clases, orígenes y limpieza de las eflorescencias en materiales cerámicos $(8,13)$

\begin{tabular}{|c|c|c|}
\hline Aspecto & Origen & Limpieza \\
\hline Polvo blanco, soluble & $\begin{array}{l}\text { Sales solubles; contaminación atmosférica; } \\
\text { ambiente marítimo }\end{array}$ & Cepillado y limpieza con agua \\
\hline $\begin{array}{l}\text { Depósito blanco (carbonato } \\
\text { cálcico) pegajoso, con } \\
\text { formación de gotas, } \\
\text { efervescente e insoluble } \\
\text { en ácido }\end{array}$ & $\begin{array}{l}\text { Carbonatación del hidróxido } \\
\text { de calcio debida a la lixiviación } \\
\text { de compuestos presentes en materiales } \\
\text { con base de cemento }\end{array}$ & $\begin{array}{l}\text { Cepillado y limpieza con una disolución de } \\
\text { ácido clorhídrico (a una concentración } \\
\text { del } 5 \text { al } 10 \% \text { y al } 2 \% \text { en juntas coloreadas); } \\
\text { en el caso de cerámica vidriada, es preciso } \\
\text { realizar pruebas previas }\end{array}$ \\
\hline
\end{tabular}


causan el deterioro del revestimiento, pero sobre todo del material de juntas $(15,16)$.

\subsection{Modificación de las condiciones iniciales (C-F)}

El empleo de material cerámico inadecuado en paredes y suelos puede llevar a defectos superficiales durante su vida útil, como son el desgaste, el rayado y las manchas (14). Si no se reparan, dichos defectos pueden conducir al desprendimiento del material a medio plazo.

En la mayor parte de los casos, este tipo de acciones son el resultado de un cambio de la naturaleza o intensidad de utilización de los espacios en que se ubica el material cerámico. Un cambio de naturaleza ocurre, por ejemplo, cuando se convierte una vivienda en una oficina, dando lugar a un cambio de las cargas de uso que ha de soportar el revestimiento cerámico de paredes y suelos. Otra modificación de las condiciones iniciales consiste en el empleo prematuro del pavimento, con el consiguiente aumento de la fisuración y el riesgo del levantamiento, además de la degradación del material de juntas. La funcionalidad de éstas disminuye en dichas circunstancias al no haberse transcurrido el tiempo de fraguado necesario (14).

\subsection{Matriz de correlación de los defectos y sus causas eventuales}

A fin de comprobar la contribución de cada causa probable a la incidencia de los defectos de junta, se elaboró una matriz de correlación, basada en la bibliografía y validada mediante la realización de 88 inspecciones de revestimientos cerámicos en paredes y suelos (5). Para rellenar las celdas de la matriz (Tabla 3), se clasificaron las posibles causas de las juntas defectuosas en causas directas (inmediatas) e indirectas (primarias). Las causas directas motivan los defectos de forma clara (p. ej., acciones ambientales, desastres naturales o provocados por la acción humana), pudiendo eliminarse mediante las técnicas de reparación adecuadas. Las causas indirectas, que necesitan de una causa directa que provoque el proceso patológico, pueden atribuirse a errores de diseño, ejecución o utilización. Dichas causas pueden prevenirse mediante la adopción de medidas adecuadas en cada fase de la vida útil del revestimiento cerámico.

En la matriz se señala, para cada defecto, las causas probables y un coeficiente que representa el grado de correlación, basada en los conocimientos actuales, entre el defecto y todas las causas observadas. Los criterios adoptados para calcular dicho coeficiente han sido (5):

- 0 - ninguna relación - ninguna relación (directa o indirecta) en absoluto entre el defecto y la causa;

- 1 ( $\square)$ - correlación baja - causa indirecta del defecto, relacionada solamente con las primeras fases del proceso de deterioro; causa secundaria del deterioro cuya presencia no es necesaria para el desarrollo de éste;

- 2 (घ) - correlación alta - causa directa del defecto, asociada con las fases finales del proceso de deterioro; cuando aparece la causa, es el motivo principal del deterioro y es imprescindible para el desarrollo del proceso.

\subsection{Matriz de correlación de los distintos defectos}

Los defectos de las juntas cerámicas pueden aparecer de manera aislada o conjuntamente. El segundo caso puede presentarse cuando hay defectos de junta ubicuos, como el Dt.j2 - cambio de color o cuando un defecto menor evoluciona hacia una degradación significativa (5). Las celdas de la matriz de correlación de los distintos defectos se han rellenado con los valores del grado de correlación basados en los criterios definidos anteriormente para la matriz de defectos vs causas probables (adaptados de (15)). Se aprecia en la Tabla 4 que los defectos que tienen grados similares de correlación con una misma causa tienen un alto grado de correlación entre si. El coeficiente representa la probabilidad de que ocurra el defecto de la columna $j$ cuando ocurre el defecto de la línea $k$.

\section{INSPECCIONES DE PARAMENTOS CERÁMICOS}

Se basaron las inspecciones de los paramentos realizadas en la anterior clasificación de los defectos de junta y sus causas probables (Tablas 1 y 3 ). El objetivo de dichas inspecciones era el de identificar los defectos de juntas más frecuentes y comprobar sus orígenes actuales. La muestra incluía los 88 paramentos cerámicos cuyas características se resumen en la Tabla 5, pertenecientes a 46 edificios situados en el Portugal peninsular y las Islas Azores.

\subsection{Frecuencia de los defectos}

Las inspecciones de los revestimientos cerámicos comprendían sólo la inspección ocular, es decir, no se realizaron pruebas in situ ni de laboratorio. Según se ve en la Figura 2, los defectos de junta (Dt.j), presentes en 70 de los paramentos de la muestra (el $80 \%$ del total), corresponden al grupo de mayor frecuencia. El gráfico también muestra la frecuencia de otros defectos, algunos que interesan a un solo elemento del revestimiento y otros a todo el paramento, concretamente: AF.1_a - falta de adhesión en la capa exterior; AF.s - falta de adhesión en el sistema cerámico; C.1_a - fisuración de la capa exterior; C.s - fisuración del sistema cerámico; Dt.t - deterioro del material cerámico; A.s - defectos estéticos del sistema cerámico. 


\begin{tabular}{|c|c|c|c|c|c|c|c|}
\hline & & 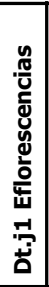 & 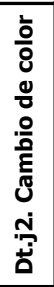 & 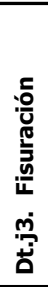 & 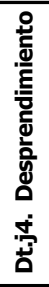 & 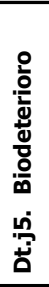 & 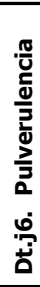 \\
\hline \multirow{6}{*}{$\begin{array}{l}\text { Errores de diseño } \\
(C-A)\end{array}$} & C-A1 elección de material incompatible, inexistente o inadecuado & $\square$ & $\square$ & $\square$ & & $\square$ & \\
\hline & C-A2 diseño de juntas incorrecto & & & $\square$ & $\square$ & & \\
\hline & C-A3 zonas de paramentos cerámicos inaccesibles a la limpieza & & $\square$ & & & $\square$ & \\
\hline & C-A4 pendiente inexistente o insuficiente en pavimentos cerámicos exteriores & $\mathbf{\square}$ & & & & $\square$ & $\square$ \\
\hline & C-A5 omisión o defecto de elementos periféricos & $\square$ & $\square$ & & & $\square$ & \\
\hline & C-A6 humedad ascendente procedente del suelo & $\mathbf{a}$ & & & & $\square$ & \\
\hline \multirow{10}{*}{$\begin{array}{l}\text { Errores de } \\
\text { ejecución (C-B) }\end{array}$} & C-B1 aplicación incompatible o no especificada del material & $\square$ & $\square$ & $\square$ & & & $\square$ \\
\hline & C-B2 instalación en condiciones ambientales extremas & $\square$ & $\square$ & & & & - \\
\hline & C-B3 incumplimiento de los tiempos establecidos entre tareas & & $\square$ & - & - & & \\
\hline & C-B4 instalación sobre fondo sucio, polvoriento o irregular & & $\mathbf{\square}$ & & & & \\
\hline & C-B5 empleo de material de agarre o lechada con fuerte tendencia retráctil & & & - & - & & $\square$ \\
\hline & C-B6 aplicación de la lechada en juntas sucias & $\square$ & $\mathbf{\square}$ & & - & & - \\
\hline & C-B7 ausencia de lechada o grosor insuficiente de la misma & & & - & - & $\square$ & $\square$ \\
\hline & C-B8 enlechado incompleto de juntas & & & & - & & \\
\hline & C-B9 pendiente inexistente o insuficiente en pavimentos cerámicos exteriores & $\mathbf{\square}$ & & & & $\mathbf{\square}$ & - \\
\hline & C-B10 empleo de accesorios metálicos no protegidos en las juntas & & $\square$ & $\square$ & & & \\
\hline \multirow{2}{*}{$\begin{array}{l}\text { Acción mecánica de } \\
\text { agentes externos (C-C) }\end{array}$} & C-C1 vandalismo / graffiti & & $\mathbf{\square}$ & & & & \\
\hline & C-C2 tráfico peatonal o rodado en pavimentos cerámicos & & - & & & & \\
\hline \multirow{9}{*}{$\begin{array}{l}\text { Acciones ambientales } \\
\text { (C-D) }\end{array}$} & C-D1 viento & $\square$ & & & & $\square$ & - \\
\hline & C-D2 irradiación solar excesiva & & $\square$ & $\square$ & $\square$ & & \\
\hline & C-D3 irradiación solar insuficiente & $\square$ & & & & $\mathbf{\square}$ & \\
\hline & C-D4 lixiviación de materiales con base de cemento & $\mathbf{\square}$ & & $\square$ & & & \\
\hline & C-D5 humectación del material cerámico & $\mathbf{\square}$ & $\mathbf{\square}$ & & & घ & - \\
\hline & C-D6 ataque biológico & & - & & & - & $\square$ \\
\hline & C-D7 contaminación atmosférica & & - & & & & $\square$ \\
\hline & C-D8 criptoeflorescencias & & & & & & $\square$ \\
\hline & C-D9 envejecimiento natural & & - & - & - & & - \\
\hline \multirow{4}{*}{$\begin{array}{l}\text { Falta de mantenimiento } \\
(\mathrm{C}-\mathrm{E})\end{array}$} & C-E1 ventilación insuficiente en interiores & $\square$ & & & & $\square$ & $\square$ \\
\hline & C-E2 falta de limpieza del paramento cerámico o de zonas adyacentes & & $\square$ & & & & \\
\hline & C-E3 limpieza incorrecta del material cerámico & & $\square$ & $\square$ & & & - \\
\hline & C-E4 defectos de fontanería/saneamiento & $\mathbf{\square}$ & & & & $\square$ & - \\
\hline $\begin{array}{l}\text { Cambio de las } \\
\text { condiciones iniciales (C-F) }\end{array}$ & C-F1 utilización prematura de los pavimentos cerámicos & & & & $\square$ & & - \\
\hline
\end{tabular}

La frecuencia relativa de cada defecto de la categoría Dt.j se indica en la Figura 3. El defecto Dt.j2 - cambio de color se halla en más de la mitad (55\%) de los revestimientos de la muestra. No obstante, dicho defecto de junta no es tan grave como los siguientes: Dt.j1 - eflorescencias, Dt.j3 fisuración y Dt.j4 - desprendimiento. Estas tres categorías de defectos producen una falta de estanqueidad en el revestimiento cerámico, reduciendo con ello su durabilidad. Se observará que el defecto Dt.j5 - biodeterioro, aun afectando a sólo un $5 \%$ de la muestra, siempre presenta un avanzado grado de degradación y aparece en zonas en que la humectación del material cerámico es excesiva.

\subsection{Frecuencia de las causas}

En la Figura 4 se muestra la frecuencia relativa de las causas probables de los defectos Dt.j. Aunque el grupo de causas C-D - acciones ambientales se asocia con casi todos los defectos de juntas observados (93\%), este resultado puede haberse influido por las características de la muestra (un $78 \%$ de los revestimientos de la muestra se ubicaban en exteriores). La asociación de los errores de diseño (C-A) con un $73 \%$ de los defectos evidencia la importancia de la fase de diseño en la prevención de situaciones patológicas. 
Tabla 4. Matriz de correlación de los defectos de las juntas cerámicas

\begin{tabular}{|lllllll|}
\hline D/D & Dt.j1 & Dt.j2 & Dt.j3 & Dt.j4 & Dt.j5 & Dt.j6 \\
\hline Dt.j1 Eflorescencias & - & $24 \%$ & $8 \%$ & $5 \%$ & $50 \%$ & $58 \%$ \\
\hline Dt.j2. Cambio de color & $17 \%$ & - & $21 \%$ & $23 \%$ & $17 \%$ & $46 \%$ \\
\hline Dt.j3. Fisuración & $10 \%$ & $37 \%$ & - & $63 \%$ & $7 \%$ & $53 \%$ \\
\hline Dt.j4. Desprendimiento & $7 \%$ & $40 \%$ & $63 \%$ & - & $7 \%$ & $57 \%$ \\
\hline Dt.j5. Biodeterioro & $63 \%$ & $30 \%$ & $7 \%$ & $7 \%$ & - & $57 \%$ \\
\hline Dt.j6. Pulverulencia & $38 \%$ & $41 \%$ & $28 \%$ & $29 \%$ & $29 \%$ & - \\
\hline
\end{tabular}

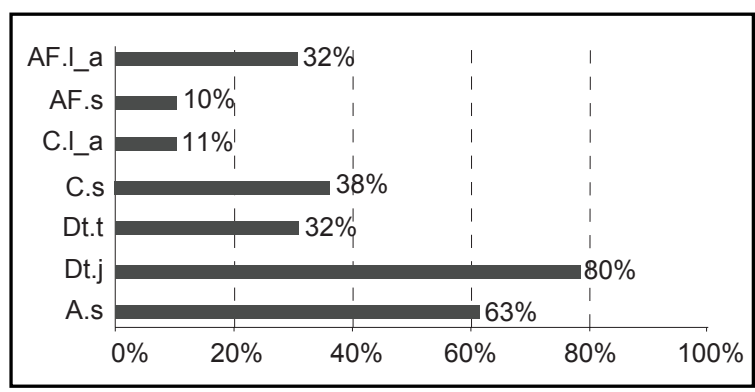

Figura 2. Frecuencia relativa de las distintas categorías de defectos detectadas en los 88 paramentos cerámicos inspeccionados.

A continuación se analizó la contribución de las causas a la incidencia de las varias categorías de defectos Dt.j (causas con una frecuencia relativa superior al $20 \%$ y defectos con una frecuencia relativa superior al 10\%). EI resultado en Dt-j1 - eflorescencias, Figura 5, es el esperado: es decir, existe una influencia directa de las acciones ambientales (C-D) en más del $90 \%$ de los casos (C-D4 - lixiviación de los materiales con base de cemento y C-D5 - humectación del material cerámico). Por otra parte, algunos de los errores de diseño causan más de la mitad de los defectos Dt.j1, contribuyendo a aumentar la agresividad de las acciones del medio.

En las Figuras 6 y 7 se aprecia la influencia de C-D5 humectación del material cerámico sobre la incidencia de los defectos de junta Dtj.2 - cambio de color y Dt.j3 - fisuración, y por lo tanto la importancia de dicha causa en la merma de las propiedades y prestaciones del material de juntas.

Otras dos causas (C-B10 - empleo de accesorios metálicos no protegidos en las juntas y C-D9 - envejecimiento natural) contribuyen al defecto Dt.j2 en más de la mitad de los casos. El defecto C-B10 indica una falta de cuidado en la elección y colocación de los accesorios de los revestimientos cerámicos. El C-D9 revela una falta de mantenimiento por parte de los propietarios del edificio. En más del $50 \%$ de los casos en que se observa el defecto Dt.j3 fisuración las causas contribuyentes son C-A1 - elección de material incompatible, inexistente o inadecuado, C-B5 - empleo de material de agarre o lechada de
Tabla 5. Características de los paramentos cerámicos (edad media: 31 años)

\begin{tabular}{|c|c|c|c|}
\hline & \multicolumn{2}{|c|}{ Paramentos cerámicos inspeccionado } & \\
\hline & Suelos & Paredes & \\
\hline Interiores & $8(9 \%)$ & $11(13 \%)$ & $19(22 \%)$ \\
\hline \multirow[t]{2}{*}{ Exteriores } & $5(6 \%)$ & $64(72 \%)$ & $69(78 \%)$ \\
\hline & $13(15 \%)$ & $75(85 \%)$ & $88(100 \%)$ \\
\hline
\end{tabular}

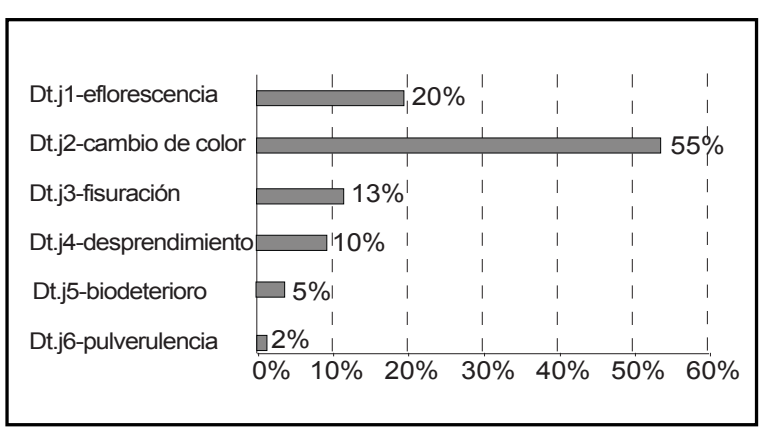

Figura 3. Frecuencia relativa de las distintas categorías de defectos Dt.j detectadas en las inspecciones.

fuerte tendencia retráctil, C-B7 - ausencia de lechada o insuficiente grosor de la misma y C-D9 - envejecimiento natural. Dichas causas indican negligencia por parte del proyectista (C-A1), del contratista (C.B5 y C-B7) y del usuario final (C-D9) en relación con las juntas, el elemento que asegura la permeabilidad del paramento al vapor de condensación a la vez que la estanqueidad, garantizando la durabilidad prevista del material cerámico.

\section{CONCLUSIÓN}

Los datos recogidos in situ permitieron constatar la importancia de los defectos de junta en una muestra representativa de paramentos con revestimiento cerámico y definir las causas más probables de su aparición. La durabilidad de los revestimientos cerámicos no es eterna y depende directamente de la fase de degradación del material de juntas, así como de su diseño, ejecución y mantenimiento. Las actuaciones preventivas, entre las que se incluyen el diseño adecuado de las juntas y la planificación de su mantenimiento, constituyen la forma de evitar la degradación de los revestimientos cerámicos durante su vida útil. Su realización es asimismo la estrategia más adecuada, desde el punto de vista tanto técnico como económico, para asegurar la funcionalidad prevista del revestimiento. Esta estrategia evita la degradación de los revestimientos cerámicos por la penetración de agentes agresivos. No debe olvidarse que las juntas son el punto crítico de la superficie de dichos revestimientos, en el que comienza la mayor parte de los procesos de degradación (9). 


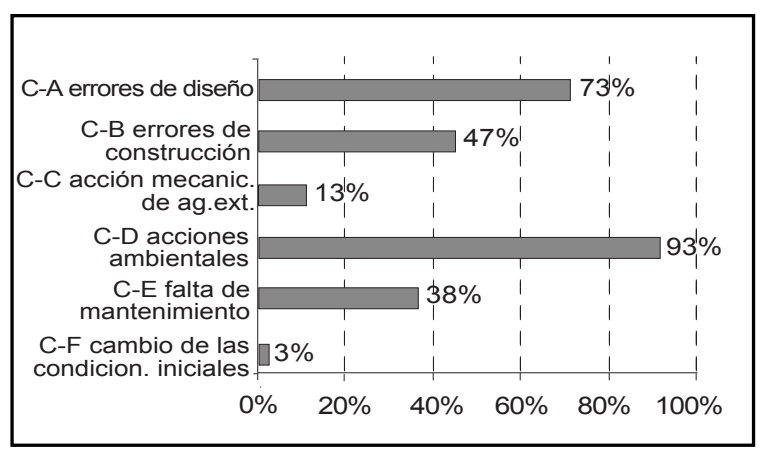

Figura 4. Frecuencia relativa de las causas probables de los defectos Dt.j.

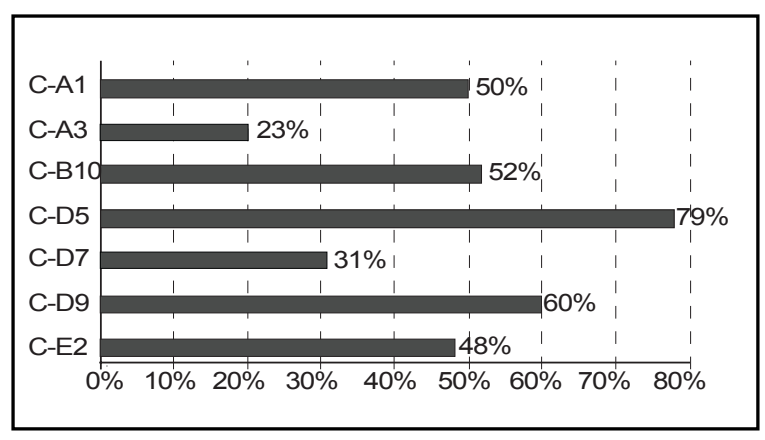

Figura 6. Causas que contribuyen a la incidencia del defecto Dt.j2 - cambio de color.

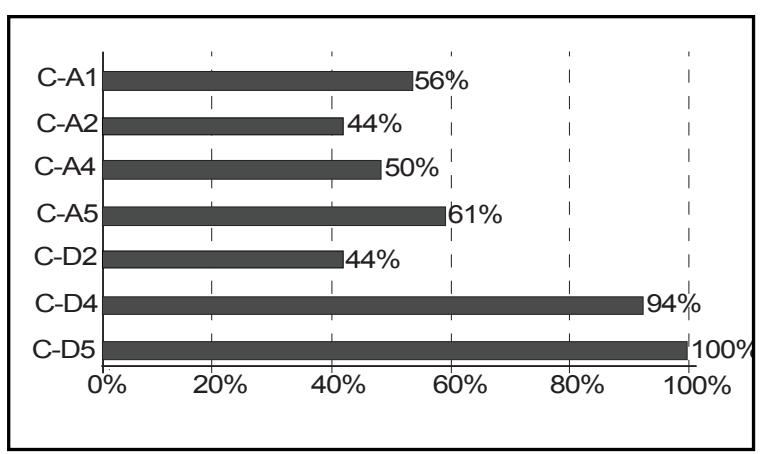

Figura 5. Causas que contribuyen a la incidencia del defecto Dt.j1 - eflorescencias.

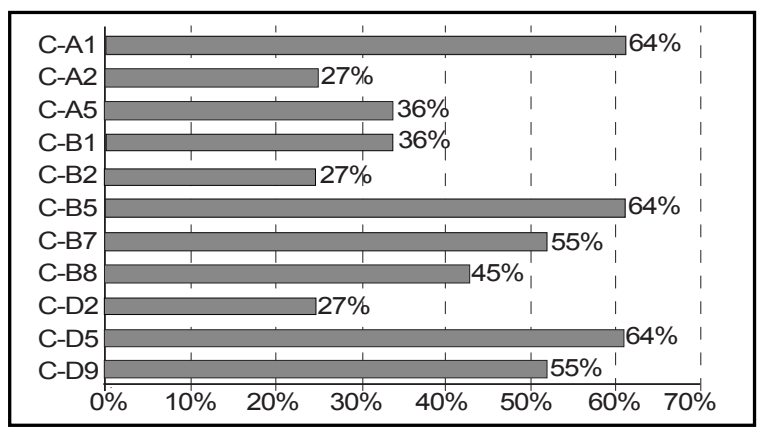

Figura 7. Causas que contribuyen a la incidencia del defecto Dt.j3 - fisuración.

\section{BIBLIOGRAFÍA / BIBLIOGRAPHY}

(1) Silvestre, J. D. y Brito, J. de: "Ceramic tiling defects classification" (in Portuguese), Construção 2004 Proceedings, Faculdade de Engenharia da Universidade do Porto, Porto, Portugal, diciembre, vol. 2 (2004), pp. 879-884.

(2) Gómez, C.: "Ceramic tiling pathologies", Qualicer 2004 Proceedings, Castellón, 2004, pp. PD 03-10.

(3) Lo, Y.: "Delamination of external wall finishes of housing", XXX IAHS World Congress on Housing -Housing Construction- an interdisciplinary task Proceedings, Universidade de Coimbra, Faculdade de Ciências e Tecnologia, Coimbra, Portugal, vol. 3 (2002), pp. 1571-1576.

(4) Wan, W.: "Tiling failures a chronic problem re-visited", Qualicer 2004 Proceedings, Castellón, 2004, pp. PGII 49-56.

(5) Silvestre, J. D.: "Development of an expert system for inspection and diagnosis of ceramic wall or floor tiling laid on mortar or adhesive" (in Portuguese), Masters thesis on Construction at Instituto Superior Técnico, Lisboa, Portugal, septiembre de 2005.

(6) Goldberg, R.: "Direct adhered ceramic tile, stone and thin brick facades manual", Laticrete International, Inc., USA, 1998.

(7) CSTB : "Revêtements de murs extérieurs en carreaux céramiques ou analogues collés au moyen de mortiers-colles - Cahier des prescritions techniques d'exécution" (in French), Cahier 3266, Centre Scientifique et Technique du Bâtiment, France, 2000.

(8) Correia, C. M. C.: "Research on facades ceramic tilling and stone adhesion problems". (in Portuguese), Masters Thesis on Minerals and industrial rocks, Universidade de Aveiro, Aveiro, Portugal, 2003.

(9) Campante, E. F.: "Methodology for diagnosis, repair and prevention of facades ceramic tiling pathology" (in Portuguese), PhD Thesis on Engineering, Escola Politécnica da USP, São Paulo, Brasil, 2001.

(10) Junginger, Max: "Ceramic tiling grouting: joint influence on panels' stability" (in Portuguese), Masters Thesis on Engineering, Escola Politécnica da USP, São Paulo, Brasil, 2003.

(11) Resende, M. M.: "Facades cladding preventive maintenance: ceramic tiling cleaning" (in Portuguese), Masters Thesis on Engineering, Escola Politécnica da Universidade de São Paulo, São Paulo, Brasil, 2004.

(12) Ferreira, T. D.: "Architecture and durability - seaside characteristics defects" (in Portuguese), Masters Thesis on Construction, Instituto Superior Técnico, Lisboa, Portugal, diciembre de 2004. 
(13) Uemoto, K. L.: "Pathology: efflorescence damages" (in Portuguese), Tecnologia de Edificações Journal, Instituto de Pesquisas Tecnológicas, Pini Editions, São Paulo, Brasil, 1988, pp. 561-564.

(14) Carvalho Lucas, J. y Abreu, Miguel: "Pathology and rehabilitation of constructions. Buildings. Adhesive ceramic tiling. Detachment" (in Portuguese), Report 367/03 - NCMC, LNEC, Lisboa, Portugal, noviembre de 2003.

(15) Brito, J. de: "Development of a management system for concrete bridges" (in Portuguese), PhD Thesis on Civil Engineering, Instituto Superior Técnico, Lisboa, Portugal, octubre de 1992. 